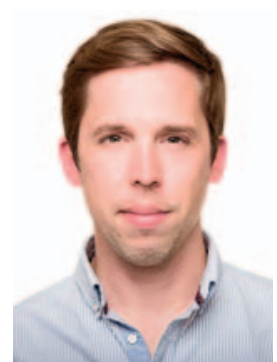

Dr. med. Matthias Raspe,

Koordination «PneumoCampus»

\section{Das Recht auf Selbtbestimmung, die Kunst der Kommunikation, das Medium der Wahl}

Auch in dieser Ausgabe des «PneumoCampus» erwarten Sie Beiträge, die sich inhaltlich von den vielen Facetten der Praxis bis hin zu den Herausforderungen in der Forschung erstrecken. Der Medizinrechtler Dr. Thomas Ruppel bietet Ihnen einen Einblick in die Komplexität von Patientenverfügungen und verdeutlicht, was junge Ärzte beachten müssen. So steht und fällt auch dieser Aspekt des Arzt-Patienten-Verhältnisses mit der richtigen Kommunikation. Die Grundprinzipien erklärt Ihnen Prof. Samy Molcho, weltbekannter Pantomime anhand der Bedeutung von Körpersprache, die im Gespräch von Arzt zu Patient, oder vielmehr von Mensch zu Mensch, aktiv erfolgreich gestaltet werden kann.

Wie erstelle ich eine erfolgreiche Veröffentlichung meiner wissenschaftlichen Arbeit? Nach einem Artikel über Tipps und Kniffe beim Schreiben eines wissenschaftlichen Artikels, stellt Ihnen in Dr. Sven Riestenpatt, Cheflektor im Karger Verlag, im zweiten Beitrag der Reihe nun Strategien zur erfolgreichen Literaturrecherche und Bewertung wissenschaftlicher Zeitschriften vor.

Ich wünsche Ihnen eine anregende Lektüre, zu deren Kritik und Anmerkungen Sie herzlich eingeladen sind!

Mit besten Grüßen,

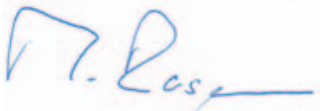

\section{KARGER}

() 2017 S. Karger GmbH, Freiburg

Fax +497614520714

information@karger.com

www.karger.com
Herrn Dr. med. Matthias Raspe

Medizinische Klinik

Schwerpunkt Infektiologie und Pneumologie

Charité - Universitätsmedizin Berlin

Augustenburger Platz 1, 13353 Berlin, Deutschland

matthias.raspe@charite.de 


\section{Medizinrecht}

\section{Patientenverfügungen - was müssen junge Ärzte beachten?}

Arzthaftungsrecht, ärztliches Arbeitsrecht, Medizinstrafrecht, ärztliches Berufsrecht und Datenschutz sind nur einige Stichpunkte aus dem Medizinrecht. Rechtsanwalt Dr. Ruppel aus Lübeck gibt Einblicke zu rechtlichen Fragen und zur Selbstständigkeit als Arzt.

\section{Jede ärztliche Maßnahme ist einwilligungsbedürftig}

Jede, auch noch so gut gemeinte, ärztliche Behandlung ist eine strafund zivilrechtlich geahndete Körperverletzung: Sie stellt ohne Einwilligung des Patienten eine Straftat dar, außerdem stehen Patienten Unterlassung-, Schadensersatz- und Schmerzensgeldansprüche zu. Bei volljährigen, bei Bewusstsein befindlichen Patienten erfolgt die Rechtfertigung dieser aus rechtlicher Sicht eigentlich zu sanktionierenden ärztlichen Eingriffe durch eine Einwilligung. Deshalb ist auch die umfassende und rechtzeitige Aufklärung so wichtig - nur so kann eine wirksame Einwilligung erreicht werden.

Kann der Patient die Einwilligung nicht geben, so ist sein mutmaßlicher Wille zu ermitteln: Was hätte der Patient gewollt, hätte man ihn fragen können?

\section{Patientenverfügung als Möglichkeit, den Willen des Patienten zu vermitteln}

Ein mittlerweile verbreitetes Instrument hierfür ist die Patientenverfügung. Sie soll, wenn sie gut abgefasst ist, den Willen des Patienten ermittelter machen. Jeder Arzt ist verpflichtet, die Patientenverfügung zu beachten, wenn sie wirksam ist. Zu ihrer Wirksamkeit muss sie schriftlich verfasst worden sein, mittels Computer reicht aus. Sie sollte aber zumindest unterschrieben sein. Auch muss der Patient bei Abfassen der Patientenverfügung noch begriffen haben, was er verfügt. Die inhaltliche Wirkung einer Patientenverfügung ergibt sich oftmals aus dem Wortlaut. Dieser ist stets ein wichtiges Indiz zu ihrer Auslegung. Jedoch darf man nicht am Wortlaut kleben bleiben. Entscheidend ist immer der Wille des Patienten, wie er sich aus dem Gesamtkontext der Patientenverfügung und ggf. auch durch Befragung der Angehörigen ergibt, selbst wenn er vom Wortlaut abweicht.

Wenn ein Patient etwa schreibt, er möchte nicht an «Maschinen angeschlossen» werden, so wollen die allermeisten Patienten durchaus auch eine Schmerztherapie, die ggf. den «Anschluss» an eine Maschine notwendig macht. Gleiches gilt etwa, wenn eine Beatmung abgelehnt wird - die meisten Patienten mit Atemproblemen dürften sich damit gegen eine Intubation entscheiden, jedoch eine Unterstützung durch eine nicht-invasive Beatmung wünschen.

Wenn der Patient einen Organspendeausweis ausgefüllt hat und die dort niedergelegte Entscheidung immer noch seinem Willen entspricht, dann haben die Angehörigen kein Mitspracherecht über die Entscheidung zur Organentnahme. Der durch die Auslegung ermittelte Wille des Patienten ist umzusetzen.

\section{Unwirksame Inhalte von Patientenverfügungen}

Manche in Patientenverfügungen geäußerten Wünsche sind jedoch unzulässig oder nicht umsetzbar: Bekanntlich ist in Deutschland die aktive Sterbehilfe verboten. Dem Verlangen aus einer Pati- entenverfügung oder von Angehörigen aktive Sterbehilfe zu leisten, ist nicht nachzukommen. Erlaubt sind hingegen die indirekte und die passive Sterbehilfe, d.h. das Sterbenlassen durch Abschalten von lebenserhaltenden Geräten sowie die Durchführung von Therapien, die als Nebenwirkung zu einem verfrühten Tod des Patienten führen, etwa bei hoch dosierten Schmerzmitteltherapien. In der Praxis untauglich und von der Rechtsprechung mittlerweile auch für unwirksam erachtet, sind Allgemeinsätze wie der Wunsch des Patienten, «in Würde zu sterben» oder ein «menschenwürdiges Leben zu führen». Niemand weiß, was der Patient hiermit wirklich sagen wollte - und kein Arzt möchte einem Patienten einen würdevollen Tod vorenthalten.

In der Krankenhauspraxis untauglich sind viele Patientenverfügungen zum Ankreuzen, weil sie kausale «Wenn-dann-Abläufe» voraussetzen, die in den allermeisten Fällen nicht gegeben sind. Insbesondere bei zum Zeitpunkt des Aufsetzens der Patientenverfügung noch mehr oder weniger gesunden Patienten und bei multimorbiden Patienten, treten die von den Ankreuz-Patientenverfügungen vorausgesetzten Kausalverläufe nicht ein. Dies ist, wenn überhaupt, nur bei austherapierten Patienten mit bestimmten Krankheiten vorstellbar. Wir empfehlen sogenannte Wunsch-Werte-Angst-Profile, d.h. nicht die Entscheidung des Patienten über einzelne, von im Zweifel überhaupt nicht zu überblickende medizinische Maßnahmen, sondern eine detaillierte «Anamnese» des eigenen Lebens, der eigenen Wünsche und der eigenen Ängste. Auf diese Weise kann in jeglichen Situationen der mutmaßliche Wille des Patienten wirklich ermittelt werden.

\section{Patienten mit Vorsorgevollmachten}

Juristisch einfach ist die Entscheidung, wenn eine Vorsorgevollmacht als Generalvollmacht besteht, die einem Angehörigen oder Freund auch die Möglichkeit einräumt, in medizinischen Inhalten zu entscheiden. Viele Vorsorgevollmachten beinhalten diese Erlaubnis jedoch gerade nicht, sondern räumen eine Vertretungsmacht in Pflegeangelegenheiten ein. Hier müssen Sie die Vorsorgevollmacht sorgsam lesen.

Selbst wenn die rechtlichen Fragen damit gelöst sind, sind solche Vorsorgevollmachten oftmals inhaltlich wenig hilfreich: Denn welchen Willen der Patient gehabt hätte, lässt sich aus einer Vorsorgevollmacht, die nur einem Dritten das Recht zu entscheiden einräumt, nicht herauslesen. Deshalb ist es im Gespräch mit dem Vorsorgebevollmächtigten unerlässlich, auch hier zwischen dem Willen des Bevollmächtigten und des Vollmachtgebers (d.h. des Patienten) streng zu unterscheiden.

\section{Vorgehen bei unwirksamen oder fehlenden Patientenverfügungen} Besteht keine Patientenverfügung, ist diese unwirksam oder unklar, kann der mutmaßliche Wille des Patienten auch durch die Befragung von Angehörigen oder Freunden herausgefunden werden. Dabei ist jedoch stets zu beachten, dass die von den Interessen des Patienten möglicherweise abweichenden Interessen der Angehö- 
rigen sorgfältig zu unterscheiden sind. Es kommt nicht auf das Interesse der Angehörigen an, sondern auf das des Patienten.

Besteht keine wirksame Patientenverfügung und hat der Arzt den Eindruck, dass die Angehörigen eher den eigenen Willen als den des Patienten formulieren, so empfiehlt es sich, den Betreuungsrichter am Amtsgericht zu informieren und eine Betreuung (früher: Vormundschaft) des Patienten anzuregen. Der Richter kommt dann in das Krankenhaus. Liegen die gesetzlichen Voraussetzungen vor, entscheidet der dann bestellte Betreuer über den Fortgang und den Abbruch von medizinischen Maßnahmen.

Kontaktadresse: Rechtsanwälte Dr. Ruppel, Kanzlei für Medizinrecht und Gesundheitsrecht, Eschenburgstraße 21, 23568 Lübeck, kanzlei@gesundheitsrecht.de, www.gesundheitsrecht.de

\section{Originalquelle:}

Ruppel T: Patientenverfügungen - was müssen junge Ärzte beachten?; in Operation Karriere - Das Karriereportal und der Kongress für angehende und junge Ärzte, www.operation-karriere.de. (Direktlink für Onlineversion: http://www.operationkarriere.de/karriereweg/von-beruf-arzt/patientenverfuegungen-was-muessenjunge-aerzte-beachten.html)

\section{operanor KARRIERE}

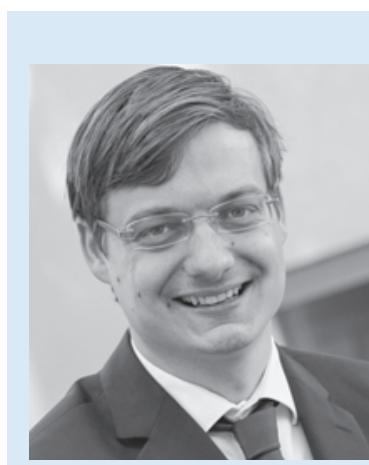

Dr. Thomas Ruppel, Lübeck

\section{Zum Autor}

Dr. Thomas Ruppel ist Rechtsanwalt für Medizinrecht in Lübeck. Er studierte Rechtswissenschaften an der Universität Greifswald. Bereits während des Studiums richtete er seinen Fokus auf das Medizinrecht. Seinen juristischen Vorbereitungsdienst absolvierte er am Landgericht in Düsseldorf, bei einer Kassenärztlichen Vereinigung und mittelständischen medizinrechtlichen Kanzleien, die ausschließlich Ärzte und andere Leistungserbringer im Gesundheitswesen beraten. Dr. Ruppel promovierte im Bereich der Versorgungsforschung und war unter anderem für eine internationale Rechtsanwaltskanzlei im Bereich Medizinrecht, Arzneimittelrecht und Medizinprodukte tätig.

Im Jahr 2012 absolvierte Dr. Ruppel den Fachanwaltslehrgang für Medizinrecht. Er veröffentlicht regelmäßig in der juristischen und ärztlichen Fachpresse.

\section{Interview mit Prof. Samy Molcho \\ Von Mensch zu Mensch statt von Arzt zu Patient}

Samy Molcho zählt zu den bedeutendsten Pantomimen des 20. Jahrhunderts und hat die Kunst der Pantomime erstmals um rein psychologische und dramaturgische Elemente erweitert. Seit seinem 10. Lebensjahr ist Molcho mit der Kunst der Darstellung und Interpretation vertraut und nennt die Bühne sein Zuhause. Er studierte klassischen, modernen und fernöstlichen Tanz sowie die Technik der Pantomime in Israel und war seither in beiden Bereichen weltweit sehr aktiv. Das Bewusstsein für die Bedeutung von Körpersprache vermittelt er in entsprechenden Seminaren, die seit Jahrzehnten großen Zuspruch finden.

Sie sind einer der bedeutendsten Pantomimen des 20. Jahrhunderts, bieten aber jetzt Coachings zu Körpersprache für diverse Berufsgruppen an. Bitte skizzieren Sie die zentralen Motive Ihrer Arbeit.

Einer der wichtigsten Aspekte einer zwischenmenschlichen Interaktion, die es immer und überall gibt, sei es mit der Familie, in der Arbeit, oder zwischen Arzt und Patienten, ist der Informationsaustausch. Da-

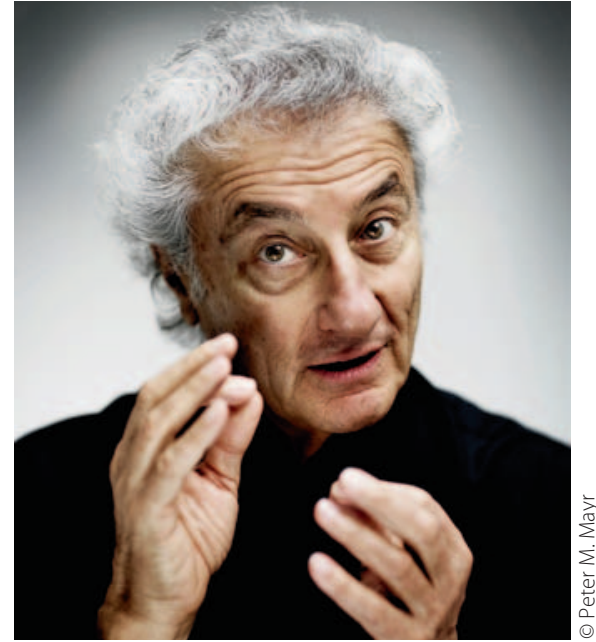

«Respektiere den Patienten zuerst als Mensch und verbessere die zwischenmenschliche Kommunikation.» lieren, was wir sagen und was wir nicht sagen wollen. Nicht so bei der Körpersprache. Oft senden wir durch unseren Körper und unsere Mimik unbewusste Informationen, die der Empfänger richtig oder falsch interpretieren kann - das kann die Kommunikation negativ beeinflussen. Durch das bewusste Einsetzen der Körpersprache erzielen wir eine höhere Wirkung und erwecken Vertrauen in unseren Gesprächspartnern. Es ist wichtig zwischen «wie wirke ich» und «was meine ich» zu unterscheiden. Die Wirkung von Signalen ist oft anders als gemeint.

Welche Rolle spielt Körpersprache in unserer Welt und welche Bedeutung hat Ihre Arbeit für die Gesellschaft im Allgemeinen?

Der Körper sendet ununterbrochen Informationen, ob wir wollen oder nicht. Unabhängig von gesellschaftlichen Spielregeln von Verhalten und Kommunikation werden innere Empfindungen, die nicht immer Hand in Hand mit diesen Spielregeln gehen, trotzdem gesendet. Sie besser zu verstehen und zu beherrschen, verbessert das zwibei ist nicht nur wichtig, was der Informationssender von sich gibt, sondern auch wie es auf den Empfänger wirkt und wie er es interpretiert. In der verbalen Kommunikation können wir mehr kontrol- schenmenschliche Verständnis und dient damit einem besseren und harmonischeren Zusammenleben in unserer Welt. Dazu verhilft uns auch die Kenntnis der Bedeutung von Signalen der Körper- 
sprache in verschiedenen Kulturen und Nationen. So werden Missverständnisse vermieden und mehr Respekt anderen Kulturen gegenüber geschaffen.

\section{Was ist bei der Körpersprache im Arzt-Patienten-Gespräch wichtig?}

Auf der medizinischen Ebene gelten nur Symptome und Wissenschaft, dabei kommunizieren aber auch sowohl der Patient als Mensch als auch der Arzt als Mensch. Eine bessere Verständigung auf der zwischenmenschlichen Ebene hilft auch bei den medizinischen Diagnosen und Behandlungen.

Kann man bei der Körpersprache «Fehler» machen? Wenn ja, was wären Beispiele?

Fehler kann man nicht machen, man kann aber unbewusst negative Signale senden. Beispiele:

- eine unzufriedene Miene wegen eines Schreibfehlers in der Akte, was der Patient aber auf sich/seine Diagnose bezieht;

- dominante Befehl-Bewegung mit dem Zeigefinger von oben nach unten;

- Vermeidung von Blickkontakt während eines Diagnosegesprächs;

- den Blick auf den Bildschirm zu richten statt auf den Patienten ist eine Missachtung des Patienten, der sich nicht wahrgenommen fühlt.

Weitere Beispiele erfahren Sie in meinen Seminaren.

\section{Worauf sollten vor allem praxisunerfahrene Ärzte achten?}

Darauf sollten auch praxiserfahrene Ärzte achten: In der Kommunikation mit Patienten von Mensch zu Mensch und nicht von Arzt zu Patient agieren. Kenntnisse der Körpersprache dienen zur Verbesserung dieser Beziehung.

Gibt es einen grundlegenden Rat, den sie jungen Ärzten mitgeben können?

Respektiere den Patienten zuerst als Mensch und verbessere die zwischenmenschliche Kommunikation, denn dadurch entstehen Sympathien, die helfen, das Vertrauen zwischen Arzt und Patienten zu fördern, was sich im Endeffekt positiv auf die Genesung des Patienten auswirkt.

\section{Prof. Molcho, vielen Dank für das Interview!}

Kontaktadresse:, Joram Harel Management, Colloredogasse 32, 1180 Wien, Österreich, office@harel.at

\section{S. Karger Verlag für Medizin und Naturwissenschaften $\mathrm{GmbH}$ Literaturrecherche, bibliographische Dienste und Kenngrößen wissenschaftlicher Qualität}

Jeder Wissenschaftler steht bei der Erstellung eigener wissenschaftlicher Studien auf den Schultern von Riesen, will heißen, seine eigene Arbeit baut auf dem auf, was tausende andere vor ihm erforscht und bestimmt haben. Und die Erkenntnisse, die er aus seinen Forschungen zieht, müssen im Kontext bereits vorliegender Befunde bestehen. Doch wie findet man geeignete Literatur für seine Arbeit?

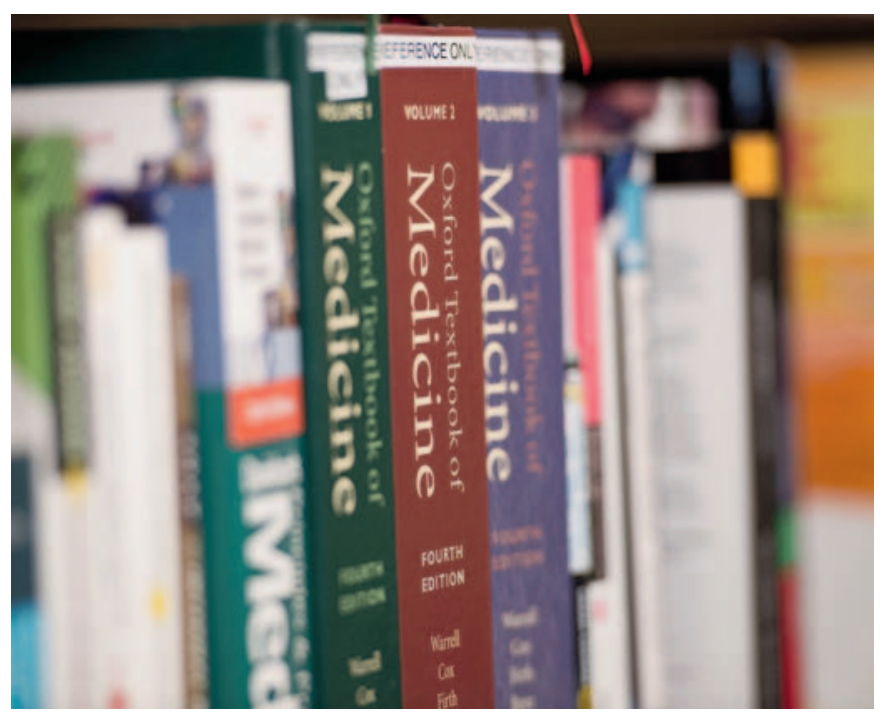

\section{Literaturrecherche}

Wie so vieles ist auch die Literaturrecherche mittlerweile im Wesentlichen internetgestützt. Ein erster Zugang ist die Literatursuche mithilfe klassischer Suchmaschinen wie Google (www.google.de) oder Bing (www.bing.de), die allerdings zu sehr unspezifischen Suchergebnissen führt. Eine deutliche Verbesserung stellt hier schon die Verwendung von Google Scholar (https://scholar.google.de) dar, bei der die Suche auf wissenschaftliche Literatur beschränkt ist. Für medizinische Fachliteratur empfiehlt sich allerdings die Verwendung spezieller medizinischer Literaturdatenbanken. Die wahrscheinlich bekannteste und am meisten genutzte dieser Datenbanken ist PubMed (www.ncbi.n/m.nih.gov/pubmed). PubMed wird von der US National Library of Medicine und dem National Institutes of Health betrieben. Sie umfasst ausschließlich englischsprachige Literatur. Zudem ist sie fokussiert auf den angelsächsischen Raum, was in Hinblick auf die Betreiber auch nicht verwundern kann. Obwohl sie sehr umfangreich ist, sind bei weitem nicht alle weltweit verfügbaren Journals darin enthalten. Neben ihrer schieren Größe macht diese Datenbank auch ihre Benutzerfreundlichkeit in der Bedienung so beliebt. So lassen sich sehr detaillierte Suchroutinen aufbauen und abspeichern, um sie zu einem späteren Zeitpunkt erneut zu verwenden. Ein auf der PubMed-Seite verfügbares Video-Tutorial (http://yzu.be/dncRQ1cobdc) führt in einfacher Weise in die Verwendung der erweiterten Suchfunktionen von PubMed ein. 
Eine weitere Datenbank, die jeder Mediziner kennen sollte, ist die Cochrane Library. Diese Datenbank umfasst Informationen von hoher Qualität, basierend auf Publikationsformen, die für evidenzbasierte Medizin unverzichtbar sind. Sie wird gepflegt von der Cochrane Collaboration, einem globalem unabhängigem Netzwerk von praktisch tätigen Ärzten, Forschern und Patientenfürsprechern [1]. Obwohl viele der dort verfügbaren Referenzen auch über PubMed auffindbar sind, empfiehlt sich eine separate Suche, da sie, anders als PubMed, kritisch geprüfte Inhalte und darüber hinaus auch Zitationen von primären Datenbanken bietet.

Für eine detaillierte Literatursuche empfiehlt sich somit der Einsatz mehrerer Datenbanken. Je nach Fragestellung ist die eine oder andere besser geeignet, wie der Artikel von Metzendorf et al. [2] eindrucksvoll zeigt.

Eine weitere bedeutende wissenschaftliche Datenbank ist Thomson Reuters Web of Science, die allerdings nicht frei zugänglich ist.

\section{Bewertung wissenschaftlicher Journals und wissenschaftlicher Arbeit}

Als Leser wird man schnell feststellen, dass es große Qualitätsunterschiede zwischen verschiedenen Zeitschriften gibt. Und als Autor wird man sich Gedanken machen, wie gut der eigene Artikel ist, um anhand dessen zu entscheiden, bei welcher Zeitschrift man seinen Artikel einreicht. Je höher das Renommee eines Journals, desto höher das Prestige einer Publikation, aber desto höher auch die Wahrscheinlichkeit, dass ein Artikel nicht zur Publikation angenommen wird und im Begutachtungsverfahren scheitert.

Der wahrscheinlich nach wie vor bedeutendste Indikator für das Renommee einer Zeitschrift ist der vom Thomson Reuters Web of Science ermittelte Impact Factor (IF). Der aktuelle IF berechnet sich wie folgt: Zitate im Jahr 2015 von Publikationen der Jahre $2013+2014$ / Anzahl der Publikationen in $2013+2014$. Für ein noch nicht abgelaufenes Jahr kann es noch keinen IF geben. Der IF 2016 ist erst Mitte des Jahres 2017 verfügbar. Jede Zeitschrift ist einem bestimmten Themenbereich zugeordnet (z.B. «Hematology»), und die Journals werden gemäß ihrem IF in einer Rangliste eingestuft. Natürlich macht es nur Sinn, Zeitschriften eines Themenbereichs zu vergleichen. Der IF ist mittlerweile ein veritabler Wirtschafts- und Einflussfaktor: Bibliotheken orientieren sich bei der Auswahl ihres Zeitschriftensortiments am IF, Forschungsinstitutionen werden anhand des IF ihrer Publikationen bewertet, die Vergabe von Fördermitteln orientiert sich am IF, Wissenschaftler beurteilen die Qualität ihrer Publikationen anhand des IF und streben die Publikation in Journals mit möglichst hohem IF an.

Eine weitere Messgröße ist der sogenannte Hirsch-Index (h-Index). «Der h-Index ist eine Kennzahl für das weltweite Ansehen eines Wissenschaftlers in Fachkreisen. Die Kennzahl basiert auf bibliometrischen Analysen, d.h. auf Zitationen der Publikationen des Wissenschaftlers. Ein hoher h-Index ergibt sich, wenn eine erhebliche Anzahl von Publikationen des Wissenschaftlers häufig in anderen Veröffentlichungen zitiert ist» [3]. Der h-Index ist stark abhängig von der verwendeten Datengrundlage. Die gängige Datenbasis ist Thomson Reuters Web of Science, die allerdings den Nachteil hat, dass Buchbeiträge nicht erfasst werden, was je nach Forschungsschwerpunkt ein schwerwiegender Nachteil sein kann. Auch andere Datenbanken wie Scopus (www.elsevier.com/solutions/scopus) oder soziale wissenschaftliche Netzwerke wie ResearchGate (www.researchgate.net) oder Mendeley (www.mendeley.com) operieren mit einem h-Index.

Kontaktadresse: Dr. Sven Riestenpatt, Projektmanagement \& Editorial Office Transfusion Medicine and Hemotherapy / Obesity Facts, S. Karger Verlag für Medizin und Naturwissenschaften GmbH, Wilhelmstraße 20a, 79098 Freiburg, Deutschland, s.riestenpatt@karger.com

\section{Literatur}

1 Timmer A, Richter B: Systematische Übersichtsarbeiten zu Fragen der Therapie und Prävention: Eine Einführung in Frage und Antwort. Teil 4 - Cochrane und die Cochrane Collaboration. Arzneimitteltherapie 2008;26:376-379.

2 Metzendorf M-I, Schulz M, Braun V: All information is not equal: using the literature databases PubMed and the Cochrane Library for Identifying the evidence on granulocyte transfusion therapy. Transfus Med Hemother 2014;41:364-374.

3 Wikipedia: h-Index. https://de.wikipedia.org/wiki/H-Index (Zugriff 02.03.2017).

Mit Bezug auf den ersten Beitrag dieser Reihe zum wissenschaftlichen Publizieren (Karger Kompass Pneumol 2017;5:41) möchten wir auf Angebote von Einrichtungen und Universitäten sowie Arbeitskreisen diverser Gesellschaften hinweisen, die entsprechende Kurse durchführen. 